\title{
DIE LEISTUNG DES LEHRERS UND DAS VERHÄLTNIS VON AFFEKTIVITÄT UND LERNEN AUS DER PERSPEKTIVE VON PIAGET UND WALLON
}

\section{REVIEW ARTICLE}

DIAS, Adailton di Lauro ${ }^{1}$

DIAS, Adailton di Lauro. Die Leistung des Lehrers und das Verhältnis von Affektivität und Lernen aus der Perspektive von Piaget und Wallon. Revista Científica Multidisciplinar Núcleo do Conhecimento. Ano 04, Ed. 07, Vol. 09, S. 64-71. Juli 2019. ISSN: 2448-0959

\section{ZUSAMMENFASSUNG}

Die Studie befasst sich mit dem Thema des Verhältnisses von Affektivität und Lernen. Er verweist auf die Bedeutung der Leistung des Lehrers in diesem Zusammenhang. Es befasst sich damit, dass Affektivität, Kognition und Motivation bei der Entwicklung des Lernens des Schülers durch Lehrervermittlung in der täglichen Schule zusammenarbeiten. Es basiert auf Theoreten wie Piaget und Wallon. Es hat als allgemeines Ziel, die Beziehung zwischen Lernen und Affektivität aus der Perspektive von Piaget und Wallon zu verstehen. Es bringt ein Forschungsproblem: Wie können Affektität, Kognition und Motivation bei der Entwicklung des Lernens des Schülers mit der Vermittlung des Lehrers im Klassenzimmer zusammenarbeiten? Aus den durchgeführten Studien geht hervor, dass aus Piagets Sicht in seiner Piagetschen Theorie Affektivität, Kognition und Motivation notwendige Voraussetzungen für die Konstitution von Intelligenz und sinnvollem Lernen sind. Nach Wallons Ansicht, in seiner Theorie namens Wallonisch, zum Lernen, ist es notwendig, zwischen funktionellen Sätzen (Affektivität, Kognition, Motricity und Person) zu integrieren.

\footnotetext{
${ }^{1}$ Mestrando em Ciências da Educação (Grendal University). Especialista em Língua Inglesa (FIJ). Graduado em Letras (UNEB). Graduado em Português e Inglês pela Universidade Metropolitana de Santos - SP. Professor EBTT Port/Ing - IFRR.
} 
Daher ist es notwendig, die Bedeutung der etablierten Beziehungen mit den emotionalen Aspekten der Schüler im Lehr- und Lernprozess zu betonen. Und dass Beziehungen mit Affektivität sich gegenseitig in Ihrem Verhalten beeinflussen können, indem sie Ihnen helfen, Wissen leichter zu erwerben, es intelligenter machen, es vorantreiben und zu Aktionen motivieren, die das Lernen beinhalten. Bedeutende. So helfen Affektivität, Kognition und Motivation bei der Entwicklung von Lernenden im Schulalltag.

Stichworte: Zuneigung, Lernen, Lehrer, Piaget, Wallon.

\section{EINFÜHRUNG}

Die Studie diskutiert den Zusammenhang zwischen Lernen und Affektivität und hebt in diesem Prozess die Bedeutung der Leistung des Lehrers im Klassenzimmer hervor und zeigt, dass sein emotionaler Zustand sowie der der Schüler positiv oder negativ im Lernen reflektiert werden. Es bringt auch das Thema Der Affektivität, Kognition und Motivation als primäre Faktoren für die Entwicklung und das Lernen der Schüler, aus der Perspektive von Piaget und Wallon.

Das Problem des Studiums versucht die Frage: Wie können Affektität, Kognition und Motivation bei der Entwicklung des Lernens des Schülers mit der Vermittlung des Lehrers im Klassenzimmer zusammenarbeiten? Um das allgemeine Ziel zu erreichen, das darin besteht, die Beziehung zwischen Lernen und Affektivität aus der Perspektive von Piaget und Wallon zu verstehen und auch auf das Problem dieser Studie zu reagieren, wurde bibliographische Forschung verwendet. Die Überlegungen über den Zusammenhang zwischen Lernen und der Entwicklung der Studierenden motivierten den Aufbau dieser Arbeit. 


\section{DIE ZUNEIGUNG, KOGNITION UND MOTIVATION ALS HAUPTFAKTOREN FÜR DIE ENTWICKLUNG UND DAS LERNEN DES SCHÜLERS}

Affektivität, Kognition und Motivation sind wesentliche Faktoren für die Entwicklung und das Lernen der Schüler in der täglichen Schule, einschließlich der Entwicklung von Intelligenz, da,

(...) ohne Zuneigung gäbe es kein Interesse, keine Notwendigkeit, keine Motivation; und folglich würden nie Fragen oder Probleme gestellt werden, und es gäbe keine Intelligenz. Affektivität ist eine notwendige Bedingung in der Konstitution der Intelligenz (PIAGET, 1992, S. 32).

Westliche Bildung hat als kulturelles Erbe, die Trennung des Mannes von seinen Emotionen, seinen Gedanken, seiner eigenen Realität, was zur Bildung schwacher Individuen des Geistes, der Energie und der emotional unwissenden Menschen führt. Dies ist also eine fragmentierte Sicht des Menschen, die bedauerlicherweise zu einem Bruch der menschlichen Beziehungen beiträgt. Diese traditionellen Dualismen des westlichen Denkens, die in der Geschichte der traditionellen Psychologie vorhanden sind, wurden jedoch von innovativen Theorien mit dem Ziel konfrontiert und in Frage gestellt, dialektisch Kognition und Affektivität, Vernunft und Emotion zu integrieren.

Die Affektivität ist jedoch für die geistige Entwicklung von Kindern und Jugendlichen unverzichtbar, sie treibt sie dazu, im Objekt des Wissens zu handeln. "Aber Affektivität ist nichts ohne Intelligenz, die innen die Mittel zur Verfügung stellt und die Enden klärt" (PIAGET, 1992, S.70). In dieser Hinsicht wirkt Intelligenz in affektiv, erheblich während des gesamten Prozesses der menschlichen Existenz, in dem tägliche Organisation zur aktiven Konstruktion, Elemente günstig für die Persönlichkeitsbildung bietet.

So ist es möglich, die Beziehungen zwischen Zuneigung und Intelligenz zu verstehen, dies entspricht den Emotionen, die sich der äußeren Welt zuwenden, orientiert an der Welt der Objekte des Studiums und der Beobachtung. Was sich Selbstintelligenz nennt, ist die Aufmerksamkeit, die das Kind und der Jugendliche beginnen, sich den 
Komponenten der Welt um sie herum zu zu leiten, um zu versuchen zu verstehen, wie die Dinge funktionieren, um sich an sie anzupassen.

Es ist die wechselseitige Wechselbeziehung dieser beiden Fälle, affektive und rationale, dass die Bildung und Entwicklung der Persönlichkeit stattfindet, die hier als das Produkt der Beziehung, die zwischen dem Subjekt und der soziokulturellen Umgebung konstruiert wird, was zu einem einzigartigen Wesen, Individuum.

Piaget (1971) warnte, dass Trotz unterschiedlicher Natur Affektion und Kognition untrennbar miteinander verbunden sind, weil sie in allen symbolischen und sinnlichen Handlungen untrennbar erscheinen. Er dokumentierte das effektive Verhalten von Handeln und Denken als kognitiver Aspekt, durch mentale Strukturen und einen affektiven Aspekt, durch eine Energie, die Affektivität ist.

Vygotsky (2002) versteht den Menschen als ein Wesen, das die Fähigkeit hat zu denken, zu denken, zu denken, abzuleiten und abstrakt, aber auch als jemand, der mit Gefühlen, Emotionen, Wünschen, Phantasie und Sensibilisierung für die Außenwelt ausgestattet ist. So kann man das Gefühl und die Vernunft nicht getrennt analysieren. Vielmehr ist eine Analyse in ihrer Gesamtheit sinnvoll, weil sie auf günstige Subventionen für mögliche Lösungen für bestehende Probleme hinweist. Denn "es zeigt die Existenz eines dynamischen Sinnsystems, in dem affektive und intellektuelle zusammenkommen" (VYGOTSKY, 2002, S. 7). Es ist jedoch unerlässlich, die Bedeutung der Beziehungen zu stärken, die Pädagogen mit den emotionalen Aspekten der Schüler im Lehr- und Lernprozess herstellen.

Was die Emotionen betrifft, so sagt Wallon (apud GALV-O, 1995), dass es von Anfang des Lebens an entstanden ist; verteidigt sie als erste und stärkste Bindung zwischen Individuen. Am Anfang seiner Existenz wird das Kind allmählich zu einem soziokognitiven Wesen, wenn es allmählich baut, eine einzigartige und besondere Sicht auf seine Existenz.

Wallon (apud GALV-O, 1995) besagt auch, dass Affektivität erscheint, wenn symbolische Elemente entstehen, das heißt, es manifestiert sich aus dem Kontakt mit 
dem anderen, macht deutlich, dass Affektivität eine Reihe von Manifestationen beinhaltet, die Gefühle und Emotionen umfasst.

Im Rahmen dieser Betrachtung spricht Galvo im Text "Ausdruckskraft und Emotionen, nach Wallons Perspektive" an, dass:

Schon früh, durch seine impulsiven Gesten, Verrenkungen oder Körperkrämpfe, sowie die primitivsten emotionalen Ausdrücke, wie weinen oder lächeln, mobilisiert das menschliche Baby die Menschen seiner Umgebung in einer Art affektiver Ansteckung. Der Erwachsene interpretiert dementsprechend seine Werte, Wünsche und Erwartungen, die Bedeutung der emotionalen Ausdrücke des Babys, die dazu gebracht werden, nach seinen kulturellen Parametern, Wünschen und seinen individuellen Überzeugungen zu handeln, eingehüllt in das Klima der Ansteckung, das diesen (GALV-O, 1995, S. 74).

Auf diese Weise, die das menschliche Baby ausdrücken muss, sind jedoch Manifestationen reiner Emotionen und provozieren affektive Reaktionen, die sie dazu bringen, die Bedürfnisse des Kindes zu erfüllen. In diesem Sinne ist Affektivität das Verhalten, das den anderen beeinflussen kann, so dass es es in die Tat umsetzt. Die erste Form der Zuneigung liegt in Emotionen, deren Aufgabe es ist, Individuen durch organische und intime Reaktionen global und undifferenziert miteinander zu vereinen.

Durch diese Vereinigung beziehen sich die Schüler, wenn sie in die Schule kommen, aufeinander und gewinnen bedeutende Fortschritte im kognitiven Bereich. Damit erweitern sich affektive Bindungen und der Lehrer spielt eine wichtige Rolle bei der Entwicklung der Schüler im schulischen Kontext.

Um das Motivationsverhalten zu affektiven und kognitiven Aspekten einzuhalten, ist es erwähnenswert, dass:

Die Entwicklung von Intelligenz ermöglicht es zweifellos, Motivation durch eine wachsende Anzahl von Objekten oder Situationen zu wecken. Während dieser Entwicklung bleibt das Grundprinzip jedoch das gleiche: 
Affektivität ist die treibende Quelle der Aktionen, und die Vernunft steht ihr zur Verfügung (LA TAILLE, 1992, S. 65).

Motivation wiederum ist ein integraler Bestandteil des Lehr- und Lernprozesses, ist eines der wesentlichen und wesentlichen Elemente, um etwas zu lernen oder zu erreichen. Alle Aktionen werden von einer Motivationskraft bewegt. "Motivation ist die treibende Kraft des Verhaltens. Es ist die innere Bedingung, die den Einzelnen aktiviert und inn dazu verleitet, bestimmte Antworten zu geben" (CORIA-SABINI, 2004, S. 83).

Um jedoch zu lernen oder zu lehren, muss eine motivierende treibende Kraft auftreten. Das Fehlen dieses täglichen Unterrichts in der pädagogischen Praxis sowohl in der Studenten- als auch in der Richtung und in der Fakultät wird die kognitive Entwicklung der Studenten beeinträchtigen.

\subsection{AffEKTITÄT UND SEINE BEZIEHUNG MIT LEARNING: DIE LEISTUNG DES LEHRERS IM CLASSROOM}

Wenn man die Bedeutung des Lehrers im Klassenzimmer versteht, wird wahrgenommen, dass sein emotionaler Zustand, wie auch der der Schüler, sich im Lernen widerspiegelt. Der Lehrer, indem er Zuneigung in seinen Handlungen zeigt, motiviert den Schüler, aktiv an seinen Klassen teilnehmen zu wollen, und erkennt den Lehrer als Freund, der immer bereit ist, inm zu helfen und inn zu unterrichten.

Für Oliveira und Chadwick (2001, S. 52),

Der emotionale und affektive Zustand der Schüler beeinflusst ihr Lernen. Motivation ist wie ein Auslöser, der das Lernen antreibt und die Bedingungen festlegt, unter denen es auftritt. Emotionen spielen eine große Rolle beim Lernen in jeder Lebensphase. Aber sie sind besonders wichtig bei jüngeren Schülern, da die Schulsituation in Bezug auf ihr Leben ziemlich künstlich ist und nicht sehr kompatibel mit ihren Vorlieben ist. 
Die Praxis eines affektiven Lehrers wird sein, den Lernrhythmus jedes Schülers zu respektieren, weil er die Sensibilität hat, wahrzunehmen, wann oder nicht, um mit dem Inhalt fortzufahren. Ein Schüler braucht vielleicht mehr Zeit, um eine bestimmte Disziplin zu lernen als sein Kollege, aber es kann auch schneller sein in einer Disziplin, die mehr Affinität hat.

Wie Mendes (2017) versichert, ist es notwendig, über pädagogische Praxis und Lehrerausbildung nachzudenken. Er stellt fest, dass die Weiterbildung die Rolle des Pädagogen als Berufskraft reflektiert, aber diese Ausbildung spiegelt sich vor allem in diesem Beruf als Person, weil Der Einzelne am Ende von anderen Menschen beeinflusst wird und durch das, was sie in Entscheidungen oder Entscheidungen und was in der Schule vermittelt wird. Die von diesem Autor gewählte Theorie ist wallonisch, deren Hauptanliegen es ist, funktionelle Sets zu integrieren - Affektivität, Kognition, Motricity und die Person und auch den mittleren Organismus.

Für Wallon (2007) vermittelt dies also das Verständnis der Person in ihrer Gesamtheit und versteht sie nicht nur aus der Perspektive eines der Sets, sondern durch die kontinuierliche Integration zwischen innen. Die Studie von Henri Wallon (1999) betont auch die Umgebung, in die die Person eingefügt wird, weil sowohl das Individuum als auch die Person voneinander beeinflusst werden. Aus dieser Perspektive betrachtet Wallon dieses Element in seinem Entwicklungsprozess als ähnlich wichtig.

Damit der Lehrer eine gute Arbeit zu tun, mit dem Ziel, so viele Schüler wie möglich zu erreichen, muss er verschiedene Unterrichtstechniken verwenden, die Annahme nicht nur einzelne Werke, sondern auch in Paaren, Gruppe, etc. Darüber hinaus ist es auch wichtig, dass lehrer ein gutes Verhältnis zu anderen Lehrern in der Schule haben, um Informationen über Schüler in jedem Bereich des Wissens auszutauschen.

In diesem Zusammenhang ist der Lehrer für die Förderung von Mitteln zuständig, die Motivation gewährleisten, in denen sie zum Lernen führt. Zu diesem Coria-SabiniAdressadresse: 
Motivation hat einen zyklischen Aspekt. Erstens gibt es einen Bedarf, der sich durch spezifische innere Stimulation manifestiert. Zweitens gibt es Maßnahmen, die darauf abzielen, ein Ziel zu erreichen. Sobald dieses Ziel erreicht ist, werden die Erleichterung und der Rückgang der Spannung befolgt. Diese Erleichterung ist vorübergehend und nach einiger Zeit wird der Zyklus wieder aufgenommen. Ein Baby, das weint, beruhigt sich nach der Fütterung. Stunden später taucht jedoch der Hunger wieder auf und sollte wieder befriedigt werden (CORIA-SABINI, 2004, S. 84).

In der Tat ist dies, was im Klassenzimmer geschehen sollte, muss der Lehrer Strategien haben, die den Schüler motivieren, auf das Objekt des Wissens zu handeln; Wenn es dem Schüler gelingt, seine Schlüsse zu ziehen, wird er sich erleichtert fühlen. Daraus kommt wieder der Lehrer, der neue Problematisierungen bringt, und der Schüler wird sich wieder auf die Suche nach dem neuen Wissen machen. Jedes Mal, wenn der Schüler das vorgeschlagene Ziel erreicht, ist es An, dass der Lehrer andere Herausforderungen für den Schüler vorschlägt, um sein Potenzial weiter zu entwickeln.

In Bezug auf die Mittel oder Methoden, die der Lehrer verwendet, um seine Schüler zu motivieren, kommen nicht zu dem Fall, jeder arbeitet in einer differenzierten Art und Weise, was zählt, ist, in einer Weise zu arbeiten, dass der Schüler das Lernen als eine persönliche Leistung wahrnimmt. Da zum Beispiel ein Schüler eine bestimmte Tätigkeit ausführt, die er für unfähig hielt, sie zu erfüllen, nachdem der Lehrer ihm einen Toast angeboten hatte, der inn aufforderte, ihn auszuführen. Damit kann der Student seine Schlussfolgerungen ziehen, dass er in der Lage ist, die Aktivität durchzuführen, ohne Auszeichnungen, sondern als persönliche Leistung.

Die Ansätze der zitierten Autoren wie Piaget, Vygotsky und Wallon zeigen jedoch, dass Emotion neben Vernunft eine wesentliche Rolle beim Lernen der Schüler spielt. Ihrer Meinung nach kann sich die Arbeit des Lehrers nicht auf bloßes Auswendiglernen beschränken, sondern auf das Gefühl und den Nervenkitzel mit innen. Leider ist dieses Verständnis der Relevanz und Rolle, die Emotionen im Lehr- und Lernprozess einnehmen, unter Lehrern selten, da die Informationen, die diese Idee anführen, nicht 
weitergegeben werden und immer noch berücksichtigt werden. dass pädagogische Theorien wie der Technikismus, die der pädagogischen Praxis zugrunde liegen, diesem emotionalen Aspekt der menschlichen Entwicklung keinen gebührenden Wert verleihen.

Saltini zitiert neue Paradigmen in der Bildung, unter ihnen, es ist erwähnenswert, was es sagt:

Es wird einen Raum und eine Zeit in der Schule geben, in der ich meine Klassenkameraden, die Welt, in der ich lebe, meine Ideen, Fantasien, meine Entdeckungen, Erfindungen, Wünsche und Träume hervorheben kann. Diese, die aus unserem Kern hervorgehen, binden und machen Sinn in den Objekten der Außenwelt, das heißt, jeder in sich selbst baut seine eigene Bindung auf, schafft ihre Symbole und Werte sowie ihre besondere Bedeutung und ist durch diese gleichen Bedeutungen dass das Subjekt Verbindungen zwischen der inneren Welt schafft. Daraus und damit müssen wir eine pädagogische Methodik respektieren und schaffen, die das Thema zu seiner vollen Entwicklung führt (SALTINI, 2008, S. 31).

In diesem Sinne wird erwartet, dass die Schule zu einem Untersuchungsumfeld wird, in dem das Subjekt dieses Prozesses seine Gedankenformen, seine Zweifel und Entdeckungen zum Ausdruck bringen kann, so dass es seine kommunikative Kapazität verbessert und sich ausdehnt, signifikant, seine Einfügung in den Raum, die das Potenzial der Studenten nicht nur im Bereich der Rationalität entwickelt, sondern auch im Bereich der Emotionen.

Kurz gesagt, Piaget, neben der Priorisierung der Bedeutung der biologischen Reifung für den Erwerb des Lernens, berücksichtigte auch die Bedeutung der Interaktion für den Aufbau von Wissen. Vigotsky ließ seine ganze Theorie im Zusammenspiel zwischen Individuum und Mitte unterstützen. Damit der Austausch zwischen Gleichaltrigen effektiver ist, hob er die Rolle der Sprache für den Ausdruck des Denkens hervor. Mit dem Fokus auf die Beziehung zwischen affektiven und kognitiven 
Bereichen, Wallon erstellt die Theorie der Persönlichkeitsentwicklung, Hervorhebung, dass dies zwischen Affektivität und Kognition gebildet wird. Darüber hinaus wies er darauf hin, dass Die Affektivität eine der ältesten Entwicklungsphasen sei.

\section{FAZIT}

Die Studie betont die Notwendigkeit des Lehrers, im Klassenzimmer die Integration zwischen

affektive und kognitive Dimensionen im Lehr- und Lernprozess, da Affektivität und Kognition miteinander verbunden sind. In diesem Zusammenhang erfordert die Entwicklung der genauen Affektivität der im Geheimdienstplan durchgeführten Ausarbeitungen und auch die Entwicklung der Intelligenz die affektiven Konstruktionen.

Affektivität ist immer noch ein weites Feld für die Forschung, wurde durch diese Studie festgestellt, dass es extrem wichtig für den Bildungsprozess und vor allem eine gute Beziehung zwischen Lehrer und Schüler zu etablieren.

Leider wachsen Kinder während ihrer Entwicklung oft in einer Umgebung auf, in der die Menschen einander nicht respektieren, in Feindseligkeit leben, in dem Essfähigkeit fehlt. Die Tendenz ist, dass diese Kinder sowohl in ihren Familien als auch in der Schule aggressiv werden. Von dem Moment an, in dem Kinder aggressiv werden, werden Schwierigkeiten in sozialen Beziehungen auftreten. Daher ist es notwendig, dass der Lehrer weiß, wie man mit seinen Schülern umgeht, indem er Zuneigung in seinen Handlungen zeigt.

Der Pädagoge sollte sich jedoch bewusst sein, dass es nicht ausreicht, nur affektiv zu sein, damit der Schüler lernt. Dazu muss der Lehrer zunächst ein Forscher sein, der sich mit seiner pädagogischen Praxis beschäftigt, sich in seinem Handeln seiner sozialen und ethischen Funktion verpflichtet fühlt.

Affektivität ist nur ein Weg zur Verbesserung der Lehrer-Schüler-Beziehung, die zum Schulerfolg beider beitragen wird. Durch Zuneigung im Zeit des Dialogs zwischen allen 
Mitgliedern der Schulgemeinschaft können Zweifel ausgeräumt, Disziplinlosigkeit umgangen werden und der Lernwille der Schüler wird hoch sein und das Lernen wird erreicht.

\section{REFERENZEN}

CORIA-SABINI, Maria Aparecida. Psicologia do Desenvolvimento. 2. ed. São Paulo: Ática. 2004.

GALVÃO, Izabel. Henri Wallon: Uma concepção dialética do desenvolvimento infantil. Petrópolis, RJ: Vozes, 1995.

LA TAILLE, Yves de; OLIVEIRA, Marta Kohl de; DANTAS, Heloysa. Piaget, Vygotsky e Wallon: teorias psicogenéticas em discussão. 13. ed. São Paulo: Summus, 1992.

MENDES, Daniela Barros. Memórias afetivas: A constituição do professor na perspectiva de Henri Wallon. Sao Paulo: Loyola, 2017.

PIAGET, Jean. A equilibração das estruturas cognitivas. Problema central do desenvolvimento. Trad. Álvaro Cabral. Rio de Janeiro: Zahar, 1976.

Desenvolvimento e aprendizagem. In: BRINGUIER, J.; PIANCELLA, J.R NESS. J.S.V., Org. In: Studying teaching. 2. ed. Prentice-Hall, 1971.

SALTINI, Cláudio J. P. Afetividade e Inteligência. 5. ed. - Rio de Janeiro: Wak Editora. 2008.

VYGOTSKY, Lev Semenovich. A formação social da mente: o desenvolvimento dos processos psicológicos superiores. 6. ed. São Paulo: Martins Fontes, 2002.

WALLON, Henri. A evolução psicológica da criança. Lisboa: Edições 70, 1999. Afetividade e aprendizagem - Contribuições de Henry Wallon. São Paulo: Edições Loyola, 2007. 
Eingereicht: Juni 2019.

Genehmigt: Juli 2019. 